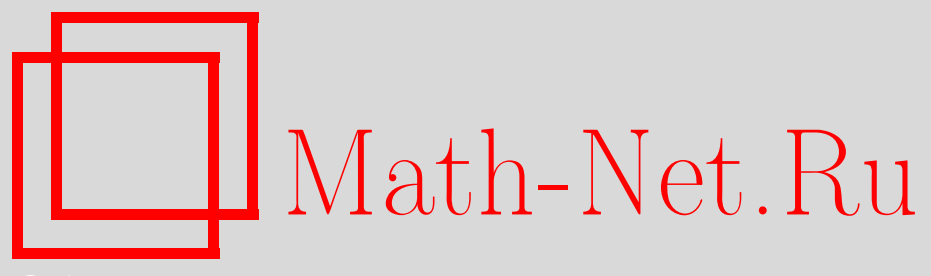

П. В. Бибиков, Относительная угловая мера конуса, сопряженного фундаментальному конусу конечной линейной группы, порожденной отражениями, УМН, 2010, том 65, выпуск 1, 179-180

DOI: https://doi.org/10.4213/rm9334

Использование Общероссийского математического портала Math-Net.Ru подразумевает, что вы прочитали и согласны с пользовательским соглашением http: //www . mathnet.ru/rus/agreement

Параметры загрузки:

IP: 54.80 .97 .219

26 апреля 2023 г., $15: 19: 46$

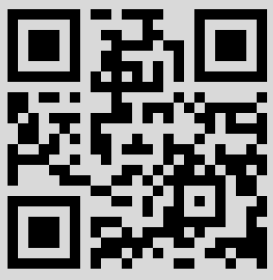




\section{Относительная угловая мера конуса, сопряженного фундаментальному конусу конечной линейной группы, порожденной отражениями}

\section{П. В. Бибиков}

Пусть $V$ - евклидово пространство со скалярным произведением $(\cdot, \cdot), W \subset O(V)$ конечная линейная группа, порожденная отражениями, $C$ - ее фундаментальный конус и $C^{\circ}-$ его открытое ядро. Через $C^{*}$ будем обозначать сопряжсенный конус, т. е. $C^{*}=\{v \in V: \forall u \in C,(v, u) \geqslant 0\}$. Будем считать, что пространство неподвижных векторов группы $W$ нулевое. Обозначим через $W_{\text {reg }}$ множество элементов из $W$, не имеющих ненулевых неподвижных векторов.

ОПРедЕЛЕНИЕ. Для любого конуса $K \subset V$ назовем его относительной угловой мерой и обозначим через $\sigma(K)$ отношение меры его пересечения с единичной сферой (с центром в нуле) к мере всей сферы.

Ясно, что $\sigma(C)=1 /|W|$. Цель настоящей заметки - дать более простое и ясное доказательство следующей теоремы.

Теорема 1 (К. Де Кончини, К. Прочези [1]). $\sigma\left(C^{*}\right)=\left|W_{\text {reg }}\right| /|W|$.

Доказательство в [1] было проведено путем перебора случаев. Общее доказательство было дано Дж. Р. Стембриджем (см. [2]), но оно было довольно сложным и косвенным. Прямое комбинаторное доказательство предложено Г. Денхамом (см. [3]). Ниже мы приведем простое геометрическое доказательство, основанное на следующем фундаментальном результате.

Теорема 2 (Ж.-Л. Вальдшпургер [4]). Имеет место равенство

$$
C^{*}=\bigsqcup_{w \in W}(1-w) C^{\circ}
$$

Простое доказательство этой теоремы см. в [5], [6].

ДоказАтельСтво теОРемы 1. Рассмотрим сопряженные конусы всех фундаментальных конусов, т. е. конусы вида $(w C)^{*}$, где $w \in W$. Их количество равно $|W|$, и они покрывают все пространство $V$. Пусть также $U=\bigcup_{w_{\mathrm{reg}} \in W_{\mathrm{reg}}} \bigcup_{w \in W}\left(1-w_{\mathrm{reg}}\right)\left(w C^{\circ}\right)$. Из теоремы Вальдшпургера следует, что $U$ - плотное открытое подмножество в $V$. Докажем, что каждый вектор $u \in U$ покрывается в точности $\left|W_{\text {reg }}\right|$ конусами вида $(w C)^{*}$. Для этого рассмотрим элемент $w_{\text {reg }} \in W_{\text {reg }}$ и сопоставим ему элемент $w \in W$ такой, что $u \in\left(1-w_{\text {reg }}\right)\left(w C^{\circ}\right) \subset(w C)^{*}$ (такой элемент единствен, так как вектор $\left(1-w_{\text {reg }}\right)^{-1} u$ лежит в открытом ядре некоторого фундаментального конуса). Это отображение задает биекцию между множествами $W_{\text {reg }}$ и $W(u)=\{w \in W$ : $\left.u \in(w C)^{*}\right\}$ : если $u \in(w C)^{*}$ для некоторого $w \in W$, то по теореме Вальдшпургера

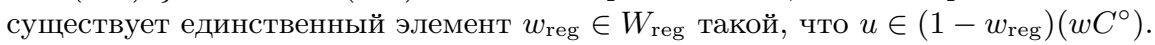

Таким образом, каждый вектор $u \in U$ покрывается в точности $|W(u)|=\left|W_{\text {reg }}\right|$ конусами вида $(w C)^{*}$. Отсюда следует, что $|W| \sigma\left(C^{*}\right)=\left|W_{\text {reg }}\right|$, что и требовалось доказать.

ЗАмечАниЕ. Из формулы Соломона (см. [7]) следует, что $|W|=\left(m_{1}+1\right) \cdots\left(m_{\ell}+1\right)$ и $\left|W_{\text {reg }}\right|=m_{1} \cdots m_{\ell}$, где $m_{1}, \ldots, m_{\ell}-$ показатели группы $W$ и $\ell=\operatorname{dim} V$. Поэтому относительная угловая мера сопряженного конуса может быть выражена через показатели группы $W$ :

$$
\sigma\left(C^{*}\right)=\frac{m_{1} \cdots m_{\ell}}{\left(m_{1}+1\right) \cdots\left(m_{\ell}+1\right)} .
$$


Пусть $W_{k}=\{w \in W: \operatorname{dim} \operatorname{ker}(1-w)=\ell-k\}$. Тогда $\sigma(C)=\left|W_{0}\right| /|W|$ и $\sigma\left(C^{*}\right)=\left|W_{\ell}\right| /|W|$. Можно предположить, что остальные отношения $\left|W_{k}\right| /|W|$ также связаны с относительными угловыми мерами некоторых конусов, причем эти конусы должны быть связаны и с $C$, и с $C^{*}$. Поэтому естественным представляется следующее определение.

Для каждой $k$-мерной грани $F$ конуса $C$ обозначим через $C_{F}$ прямую сумму $k$-мерной грани $F$ и $(\ell-k)$-мерной грани $F^{\perp}$ конуса $C^{*}$, ортогональной $F$. В частности, $C_{C}=C$ и $C_{0}=C^{*}$.

ГипотезА. $\sum_{F: \operatorname{dim} F=k} \sigma(F) \sigma\left(F^{\perp}\right)=\sum_{F: \operatorname{dim} F=k} \sigma\left(C_{F}\right)=\frac{\left|W_{\ell-k}\right|}{|W|}$ для всех $k=0, \ldots, \ell$.

Эта гипотеза обобщает теорему 1 об относительной угловой мере сопряженного конуса. Она проверена автором для всех групп ранга не больше 4 , а также для $A_{5}$.

Автор благодарит Э. Б. Винберга за постановку задачи и внимание к работе, а также В. С. Жгуна за ценные замечания.

\section{Список литературы}

[1] C. De Concini, C. Procesi, Atti Accad. Naz. Lincei Cl. Sci. Fis. Mat. Natur. Rend. Lincei (9) Mat. Appl., 17:2 (2006), 155-165. [2] J. R. Stembridge, Appendix to C. De Concini and C. Procesi paper, arXiv: math/0602279v2, 2006. [3] G. Denham, Atti Accad. Naz. Lincei Cl. Sci. Fis. Mat. Natur. Rend. Lincei (9) Mat. Appl., 19:1 (2008), 59-63. [4] J.-L. Waldspurger, J. Lie Theory, 17:3 (2007), 597-603. [5] П.В.Бибиков, В.С.Жгун, УМН, 64:5 (2009), 177-178. [6] П. В. Бибиков, В. С. Жгун, Изв. РАН. Сер. матем., 73:6 (2009), 29-38; англ. пер.: P. V. Bibikov, V. S. Zhgoon, Izv. Math., 73:6 (2009), 1101-1109. [7] L. Solomon, Nagoya Math. J., 22 (1963), 57-64.

\section{П. В. Бибиков (Р. V. Bibikov)}

Московский государственный университет им. М. В. Ломоносова

E-mail: tsdtp4u@proc.ru
Представлено Э. Б. Винбергом Принято редколлегией 15.12.2009 